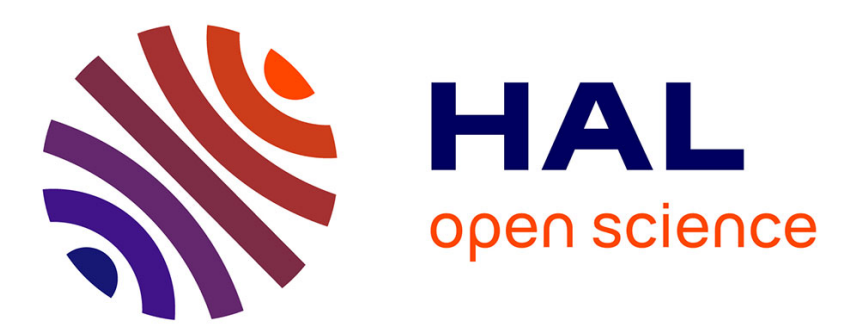

\title{
Locking-free discontinuous finite elements for the upper bound yield design of thick plates
}

\author{
Jérémy Bleyer, Canh Van Le, Patrick de Buhan
}

\section{To cite this version:}

Jérémy Bleyer, Canh Van Le, Patrick de Buhan. Locking-free discontinuous finite elements for the upper bound yield design of thick plates. International Journal for Numerical Methods in Engineering, 2015, 103 (12), pp.894-913. 10.1002/nme.4912 . hal-01184188

\section{HAL Id: hal-01184188 \\ https://hal.science/hal-01184188}

Submitted on 14 Aug 2015

HAL is a multi-disciplinary open access archive for the deposit and dissemination of scientific research documents, whether they are published or not. The documents may come from teaching and research institutions in France or abroad, or from public or private research centers.
L'archive ouverte pluridisciplinaire HAL, est destinée au dépôt et à la diffusion de documents scientifiques de niveau recherche, publiés ou non, émanant des établissements d'enseignement et de recherche français ou étrangers, des laboratoires publics ou privés. 


\title{
Locking-free discontinuous finite elements for the upper bound yield design of thick plates
}

\author{
Jeremy Bleyer ${ }^{\mathrm{a}, *}$, Canh V. Le ${ }^{\mathrm{b}}$, Patrick de Buhan ${ }^{\mathrm{a}}$ \\ ${ }^{a}$ Université Paris-Est, Laboratoire Navier, \\ Ecole des Ponts ParisTech-IFSTTAR-CNRS (UMR 8205) \\ 6-8 av Blaise Pascal, Cité Descartes, 77455 Champs-sur-Marne, FRANCE \\ ${ }^{b}$ Department of Civil Engineering, International University - VNU HCMC, Viet Nam
}

\begin{abstract}
This work investigates the formulation of finite elements dedicated to the upper bound kinematic approach of yield design or limit analysis of Reissner-Mindlin thick plates in shear-bending interaction. The main novelty of this paper is to take full advantage of the fundamental difference between limit analysis and elasticity problems as regards the class of admissible virtual velocity fields. In particular, it has been demonstrated for $2 \mathrm{D}$ plane stress, plane strain or 3D limit analysis problems that the use of discontinuous velocity fields presents a lot of advantages when seeking for accurate upper bound estimates. For this reason, discontinuous interpolations of the transverse velocity and the rotation fields for Reissner-Mindlin plates are proposed. The subsequent discrete minimization problem is formulated as a second-order cone programming (SOCP) problem and is solved using the industrial software package MosEK. A comprehensive study of the shear-locking phenomenon is also conducted and it is shown that discontinuous elements avoid such a phenomenon quite naturally, whereas continuous elements cannot without any specific treatment. This particular aspect is confirmed through numerical examples on classical benchmark problems and the so-obtained upper bound estimates are confronted to recently developed lower bound equilibrium finite elements for thick plates.
\end{abstract}

Keywords: limit analysis, yield design, upper bound, thick plates, shear locking, finite element method, second order cone programming

\section{Introduction}

Computational limit analysis generally involves the discretization of the problem fields and the use of optimization techniques to obtain sufficiently accurate bounding solutions. In

\footnotetext{
* Correspondence to: J. Bleyer, Laboratoire Navier, 6-8 av Blaise Pascal, Cité Descartes, 77455 Champssur-Marne, France, Tel : +33 (0)1 64153659

Email address: jeremy.bleyer@enpc.fr (Jeremy Bleyer)

URL: https://sites.google.com/site/bleyerjeremy/ (Jeremy Bleyer)
} 
the kinematic formulation, velocity fields must be discretized using continuous, discontinuous finite elements or discontinuities only. Owing to their simplicity and the fact that they produce strict bounds, low-order continuous displacement finite elements are widely used in numerical limit analysis procedures [1-4]. However, the accuracy of plastic limit analysis or yield design solutions is highly affected by local singularities arising from localized deformations. Therefore, there is a need to perform an adaptive refinement scheme in order to capture these local yield zones and thus improve such solutions. Moreover, volumetric locking phenomena may occur when trying to enforce the incompressibility condition in the kinematic formulations associated for instance with von Mises or Tresca yield criteria for a plane strain 2D continuum. As an alternative, "discontinuities-only" methods, such as discontinuous layout optimization (DLO), have been proposed in [5]. Instead of using finite elements, DLO problems are formulated entirely in terms of lines of discontinuity interconnecting nodes laid out across the body under consideration and defining rigid blocks. The volume being preserved by construction, the DLO numerical procedure avoids naturally the volumetric locking and can also overcome stress/velocity singularity limitations of finite element limit analysis. Yield line analysis [6] for thin plates in bending also falls into this category since the bending dissipation comes only from rotation discontinuities occurring along predefined potential yield lines. This method allows for relatively simple upper bound solutions but suffers from a certain mesh dependency and cannot, in general, produce the exact collapse load since curvature strains are not taken into account in the analysis.

Considering the advantage of both continuous and discontinuities-only methods, discontinuous elements have also been developed in parallel [7-9]. Such elements allow for velocity discontinuities across all edges shared by adjacent triangles, and consequently dissipation may occur not only inside the triangular elements but also along these discontinuities. It has been shown that discontinuous elements can give good estimates of the true collapse load with a relatively coarse mesh. Recently, Bleyer et al. [10] have proposed various types of discontinuous finite elements for plate bending, where the transversal velocity is continuous but its derivative (the rotation field) is not. The method can provide more accurate upper bound solutions compared with other element-based formulations for kinematic limit analysis of thin plate problems. Following this line of research, this paper will describe discontinuous Reissner-Mindlin elements for yield design or limit analysis of thick plates in shear-bending interaction.

It is well-known that Reissner-Mindlin plate elements exhibit shear locking phenomenon in the limit of thin plates. As a result, various methods have been proposed to overcome shear-locking effect for elastic plate problems, including selective reduced integration scheme $[11,12]$, mixed formulation/hybrid elements [13, 14], enhanced assumed strain [15], assumed natural strain [16] and discrete shear gap (DSG) formulation [17]. Shear-locking for limit analysis of Reissner-Mindlin plate has been recently studied by Le [18], where a stabilized strain smoothing technique is used in combination with DSG elements. In the present paper, a comprehensive study of the shear-locking phenomenon for yield design problems of thick plates is conducted, and it will be shown that discontinuous elements can quite naturally avoid the shear locking phenomenon. This will allow to overcome a classical drawback of dedicated procedures to remove shear-locking, in which the strain compatibility equation is 


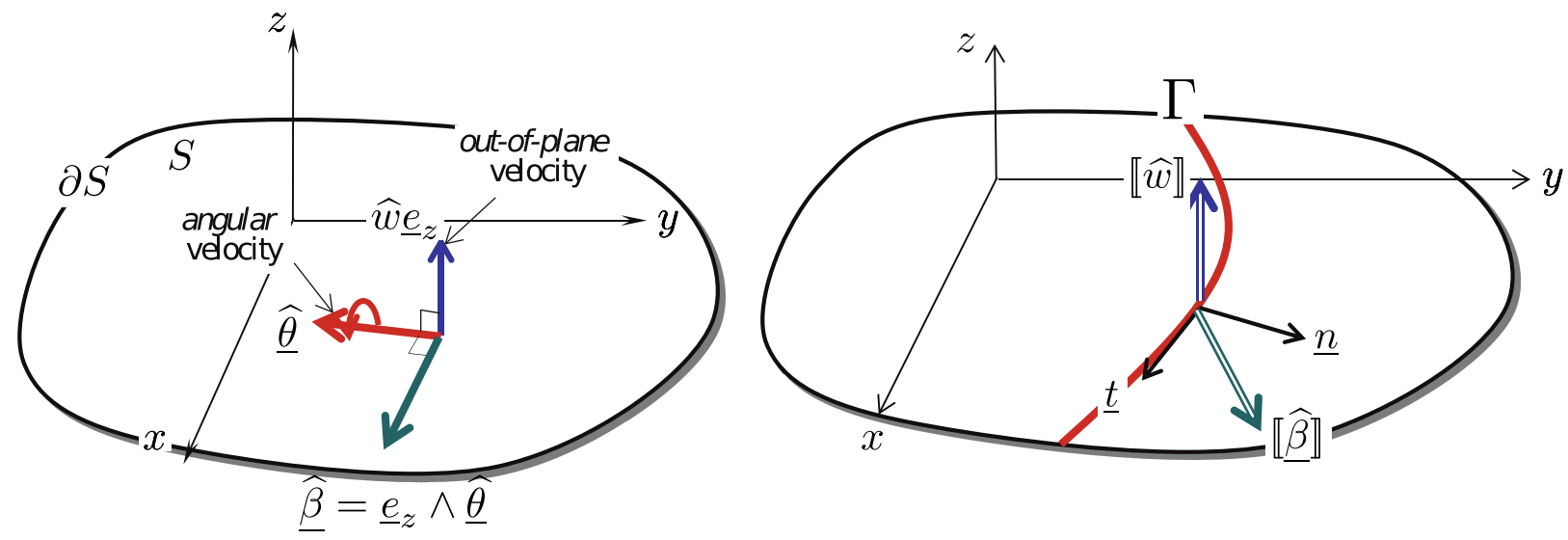

Figure 1: Virtual motions of a Reissner-Mindlin plate (left) and velocity jumps across a line $\Gamma$ (right)

often relaxed in some sense, so that the corresponding collapse load estimates can no longer be considered as true upper bounds.

The layout of the paper is as follows. Next section describes the upper bound kinematic yield design formulation for Reissner-Mindlin plate problems. Section 3 is devoted to a discussion concerning the choice of a generalized shear-bending interaction criterion for thick plates. Finite elements with discontinuous velocity fields will be introduced in Section 4 along with the corresponding second-order cone programming (SOCP) formulation. Shear locking effect will be studied in Section 5. Numerical examples are provided in Section 6 to illustrate the performance of the proposed method.

\section{Yield design of shear-bending plates}

This section recalls the yield design formulation of shear-bending plates. In this context, the plate is assumed to be infinitely resistant to membrane forces, so that the in-plane membrane strains must be equal to zero. See $[10,19]$ for more details.

\subsection{Virtual motions and virtual work of internal forces for a plate}

Referring to a Cartesian orthonormal frame Oxyz, the plate occupies a two-dimensional geometrical domain $S$ in the $O x y$-plane as shown in Figure 1. The virtual motions of such a plate are defined at any point $(x, y)$ of $S$ by:

- an out-of-plane (transversal) velocity $\widehat{w} \underline{e}_{z}$ of the particle attached to the point;

- an angular velocity $\underline{\hat{\theta}}$ representing the rotation of the microstructure attached to the same point. We note $\underline{\beta}$ the associated vector defined by $\underline{\hat{\beta}}=\underline{e}_{z} \wedge \underline{\hat{\theta}}$ which will directly appear in the expression of the shear and the curvature strains. Both vectors are parallel to $S$. 
Following the same line of reasoning as that employed for instance for one-dimensional beams, that is making use of the virtual work method and related principles, the virtual work of internal forces may be written in the form :

$$
P_{(i)}(\widehat{w}, \underline{\widehat{\beta}})=-\int_{S}\left(\underline{V} \cdot(\underline{\nabla \widehat{w}}-\underline{\widehat{\beta}})+\underline{\underline{M}}:\left({ }^{s} \underline{\underline{\nabla}}\right)\right) d S
$$

where ${ }^{s} \underline{\underline{\nabla}}(\cdot)$ denotes the symmetric part of the gradient operator with respect to the $(x, y)$ coordinates, while $\underline{V}$ is the vector of shear forces and $\underline{M}$ the tensor of bending moments. We see here that the shear strain rate is given by $\underline{\hat{\gamma}}=\underline{\nabla \widehat{w}}-\underline{\widehat{\beta}}$ and the curvature strain rate is given by $\underline{\hat{\chi}}={ }^{s} \nabla \widehat{\beta}$. In the case when the virtual velocity fields are discontinuous across a line $\Gamma$, the above expression (1) should be completed by the following additional line integral:

$$
P_{(i)}^{\llbracket \rrbracket}(\llbracket \widehat{w} \rrbracket, \llbracket \underline{\hat{\beta}} \rrbracket)=-\int_{\Gamma}((\underline{V} \cdot \underline{n}) \llbracket \widehat{w} \rrbracket+(\underline{\underline{M}} \cdot \underline{n}) \cdot \llbracket \underline{\hat{\beta}} \rrbracket) d \Gamma
$$

where $\llbracket * \rrbracket$ represents the jump of a variable $*$ when crossing $\Gamma$ along its unit normal $\underline{n}$.

\subsection{Statement of the yield design problem for shear-bending plates}

Generally speaking, the strength criterion of a plate (infinitely resistant to membrane forces) can be formulated as a condition involving all internal forces at any point of the plate, namely :

$$
f(\underline{\underline{V}}, \underline{\underline{M}}) \leq 0
$$

It is assumed from now on, that the plate loading depends upon several parameters $Q$, which means that the virtual work of external forces in any kinematically admissible (K.A.) virtual velocity field, may be expressed as:

$$
\forall(\widehat{w}, \underline{\widehat{\beta}}) \text { K.A. with } \underline{\widehat{q}}, \quad P_{(e)}(\widehat{w}, \widehat{\beta})=\underline{Q} \cdot \underline{\widehat{q}}
$$

where $\widehat{\widehat{q}}$ represents the generalized kinematic parameters defined by duality in the expression of the work of external forces. In this context, the domain $K$ of potentially safe loads $\underline{Q}$ as introduced in the yield design theory [19], is defined as follows:

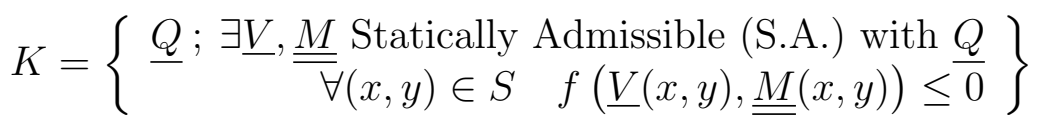

\subsection{Upper bound kinematic approach}

The upper bound kinematic approach to $K$ is then classically obtained from the virtual work principle :

$$
\begin{gathered}
(\underline{V}, \underline{\underline{M}}) \text { S.A. with } \underline{\underline{Q}} \Longleftrightarrow \\
\forall(\widehat{w}, \underline{\widehat{\beta}}) \text { K.A. with } \underline{\widehat{q}}, P_{(e)}(\widehat{\widehat{w}}, \underline{\widehat{\beta}})=\underline{Q} \cdot \underline{\widehat{q}}=-P_{(i)}(\widehat{\widehat{w}}, \underline{\widehat{\beta}})
\end{gathered}
$$


introducing the so-called maximum resisting work (also called maximum plastic dissipation in the context of limit analysis), defined as :

$$
P_{r m}(\widehat{w}, \underline{\widehat{\beta}})=\int_{S} \pi\left(\underline{\nabla \widehat{w}}-\underline{\widehat{\beta}},{ }^{s} \underline{\underline{\nabla \widehat{\beta}}}\right) d S+\int_{\Gamma} \Pi(\underline{n} ; \llbracket \widehat{w} \rrbracket, \llbracket \underline{\underline{\beta}} \rrbracket) d \Gamma
$$

where the support functions are defined as :

$$
\begin{aligned}
& \pi\left(\underline{\widehat{\gamma}}=\underline{\nabla \widehat{w}}-\underline{\widehat{\beta}}, \underline{\underline{\hat{\chi}}}={ }^{s} \underline{\underline{\nabla \hat{\beta}}}\right)=\sup _{(\underline{\underline{V}}, \underline{\underline{M}})}\{\underline{V} \cdot \underline{\widehat{\gamma}}+\underline{\underline{M}}: \underline{\underline{\hat{\chi}}} ; f(\underline{V}, \underline{\underline{M}}) \leq 0\} \\
& \Pi(\underline{n} ; \llbracket \widehat{w} \rrbracket, \llbracket \underline{\widehat{\beta}} \rrbracket)=\sup _{(\underline{\underline{V}}, \underline{\underline{M}})}\{(\underline{V} \cdot \underline{n}) \llbracket \widehat{w} \rrbracket+(\underline{\underline{M}} \cdot \underline{n}) \cdot \llbracket \underline{\widehat{\beta}} \rrbracket ; f(\underline{V}, \underline{\underline{M}}) \leq 0\}
\end{aligned}
$$

Contrary to the thin plate in bending case, we see that, in the case of a limited resistance with respect to shear forces, the transverse velocity can be discontinuous across a discontinuity line $\Gamma$. Besides the vector $\widehat{\beta}$ associated to the angular velocity can also be discontinuous, whereas only its normal component can be discontinuous in the thin plate case due to the Love-Kirchhoff condition $\widehat{\beta}=\underline{\nabla \widehat{w}}$ and Hadamard's compatibility condition [20].

As a consequence, the implementation of the yield design upper bound kinematic approach for Reissner-Mindlin plates is based upon considering distributions of K.A. transverse velocity fields $\widehat{w}$ and rotation vectors $\widehat{\beta}$ subject to the property that $\widehat{w}$ and $\widehat{\beta}$ are everywhere differentiable, except on a number of discontinuity lines where jumps of angular velocities and transverse displacement have to be taken into account.

Assuming that the plate is loaded by a transverse loading distribution $p(x, y) \underline{e}_{z}$ with a set of boundary conditions denoted by $\mathcal{B}$. The set of kinematically admissible velocity fields is $K A=\left\{\left(w, \beta_{x}, \beta_{y}\right) \in V \cap \mathcal{B}\right\}$ where $V$ is the functional space consistent with the yield design of Reissner-Mindlin plates. For this particular loading, the work of external forces writes :

$$
P_{(e)}\left(w, \beta_{x}, \beta_{y}\right)=\int_{S} p(x, y) w(x, y) d S
$$

While the maximum resisting work is $\forall\left(w, \beta_{x}, \beta_{y}\right) \in K A$ :

$$
\begin{aligned}
P_{r m}\left(w, \beta_{x}, \beta_{y}\right) & =P_{r m}^{s t r a i n}\left(w, \beta_{x}, \beta_{y}\right)+P_{r m}^{\text {disc }}\left(w, \beta_{x}, \beta_{y}\right) \\
& =\int_{S} \pi(\underline{\gamma}(x, y), \underline{\underline{\chi}}(x, y)) d S+\int_{\Gamma} \Pi(\underline{n} ; \llbracket w \rrbracket(s), \llbracket \underline{\beta} \rrbracket(s)) d s
\end{aligned}
$$

The kinematic formulation for the ultimate load factor $\lambda$ is then given by the following optimization problem :

$$
\lambda^{+} \leq \lambda^{k i n}=\min \left\{P_{r m}\left(w, \beta_{x}, \beta_{y}\right) ; \forall\left(w, \beta_{x}, \beta_{y}\right) \in K A \text { and } P_{(e)}\left(w, \beta_{x}, \beta_{y}\right)=1\right\}
$$

where $\lambda^{+}$is the ultimate value associated with the plate failure.

For the sake of simplicity, the notation ${ }^{\wedge}$ recalling the virtual character of the kinematic fields involved in the analysis will from now on be omitted. 


\section{Generalized strength criteria for thick plates in shear and bending}

\subsection{General comments}

Designing an interaction strength criterion between the shear forces and the bending moments is a particularly difficult question. For example in the case of thick beams, Drucker [21] highlighted the fact that it is impossible to obtain such a strength criterion depending only on the plane section of the beam whereas it is possible for membrane forces and bending moments interaction. Hence, such a criterion will a priori depend on an internal length scale and/or the external loading. Generalized strength criteria expressed in terms of generalized forces (or interaction diagrams) can normally be obtained when solving an auxiliary problem on an elementary volume of the underlying 3D continuum with a properly defined loading mode. The problem in the shear-bending case comes from the fact that the bending moment and the shear force are coupled from the plate equilibrium equation and cannot, therefore, be rigorously considered as two independent loading parameters (contrary to the case of the membrane force for $N$ - $M$ interaction diagrams).

For all these reasons, it is somewhat illusive to speak of an exact shear-bending strength criterion. Our approach is then based on considering a simple criterion without paying too much attention on its exactness.

\subsection{A simple strength criterion}

The strength criterion is designed such that its pure bending part reduces to a von Mises criterion for plates in bending of uniaxial bending strength $M_{0}$. Similiarly, the pure shear part is given by an isotropic criterion limiting the norm of the shear force by a value $V_{0}$ : $\sqrt{V_{x}^{2}+V_{y}^{2}} \leq V_{0}$. It is then possible to consider an interaction between those two limit cases or not [22]. In the following, we consider only a nonlinear interaction given by :

$$
G=\left\{(\underline{\underline{M}}, \underline{V}) \text { s.t. } \sqrt{\frac{M_{x x}^{2}+M_{y y}^{2}-M_{x x} M_{y y}+3 M_{x y}^{2}}{M_{0}^{2}}+\frac{V_{x}^{2}+V_{y}^{2}}{V_{0}^{2}}} \leq 1\right\}
$$

It can be observed that if $V_{0} L \gg M_{0}$ where $L$ is a characteristic in-plane length of the plate, then the previous criterion reduces to the von Mises bending criterion whereas the shear force becomes unlimited. Therefore, the situation where $V_{0} L \gg M_{0}$ corresponds to a thin plate model in which the shear strength is infinite.

As mentioned in [22], the material parameters $M_{0}$ and $V_{0}$ describing the plate strength to pure bending and pure shear respectively can be estimated by refering to a three-dimensional modelling of the plate of thickness $t$, associated with a von Mises strength criterion of ultimate tensile/compressive strength $\sigma_{0}$. In this case, $M_{0}$ and $V_{0}$ are given by the following relations :

$$
M_{0}=\frac{\sigma_{0} t^{2}}{4} \quad ; \quad V_{0}=\frac{\sigma_{0} t}{\sqrt{3}}
$$

It is to be noted that the interaction criterion (11), associated with expressions (12), has already been proposed in previous works $[23,24]$ and can be seen as an extension to plates of the strength criterion for idealized I-beams under combined bending and shear. 


\subsection{Expression of the support functions}

Remarking that (11) corresponds to an ellipsod in the 5-dimensional $(\underline{M}, \underline{V})$ space, the expression of its support function can be readily obtained as :

$$
\pi(\underline{\gamma}, \underline{\underline{\chi}})=\sqrt{\frac{4 M_{0}^{2}}{3}\left(\chi_{x x}^{2}+\chi_{y y}^{2}+\chi_{x x} \chi_{y y}+\chi_{x y}^{2}\right)+V_{0}^{2}\left(\gamma_{x}^{2}+\gamma_{y}^{2}\right)}
$$

Inspecting equation (7), it can be seen that the following relation holds true :

$$
\Pi(\underline{n} ; \llbracket w \rrbracket, \llbracket[\underline{\beta} \rrbracket)=\pi(\llbracket w \rrbracket \underline{n}, \llbracket \underline{\beta} \rrbracket \stackrel{s}{\otimes} \underline{n})
$$

where $\underline{a} \stackrel{s}{\otimes} \underline{b}=\frac{1}{2}(\underline{a} \otimes \underline{b}+\underline{b} \otimes \underline{a})$. Hence, using (13):

$$
\Pi(\underline{n} ; \llbracket w \rrbracket, \llbracket \underline{\beta} \rrbracket)=\sqrt{\frac{M_{0}^{2}}{3}\left(4 \llbracket \beta_{n} \rrbracket^{2}+\llbracket \beta_{t} \rrbracket^{2}\right)+V_{0}^{2} \llbracket w \rrbracket^{2}}
$$

\subsection{Relation to the $3 D$ continuum}

In the case of a local von Mises criterion, some authors proposed to obtain a shearbending interaction criterion by integrating through the thickness the expression of the local support function (or plastic dissipation in the context of limit analysis) in a ReissnerMindlin kinematics, that is a 3D virtual velocity field the virtual strain rate of which is of the form :

$$
\underline{\underline{d}}(z)=\left[\begin{array}{cc}
-z \underline{\chi} & \underline{\gamma} / 2 \\
\underline{\gamma}^{T} / 2 & d_{z z}(z)
\end{array}\right]
$$

where $d_{z z}(z)$ can be chosen freely as $z \operatorname{tr} \underline{\chi}$ to satisfy the divergence-free $(\operatorname{tr} \underline{\underline{d}}=0)$ condition. This approach yields the following expression ${ }^{1}[25]$ :

$$
\pi_{3 D}(\underline{\gamma}, \underline{\underline{\chi}})=\sigma_{0} \int_{-t / 2}^{t / 2} \sqrt{z^{2} \chi^{T} \mathbf{Q}_{b} \chi+\gamma^{T} \mathbf{Q}_{s} \gamma} d z
$$

where $\boldsymbol{\chi}=\left(\chi_{x x}, \chi_{y y}, 2 \chi_{x y}\right)^{T}, \boldsymbol{\gamma}=\left(\gamma_{x}, \gamma_{y}\right)^{T}$ and

$$
\mathbf{Q}_{b}=\frac{1}{3}\left[\begin{array}{lll}
4 & 2 & 0 \\
2 & 4 & 0 \\
0 & 0 & 1
\end{array}\right] \quad \mathbf{Q}_{s}=\frac{1}{3}\left[\begin{array}{ll}
1 & 0 \\
0 & 1
\end{array}\right]
$$

As stated in [26] (with a missing factor $\frac{1}{2}$ ), expression (16) is often approximated using a $n g$-points Gauss quadrature over the half thickness, so that:

$\pi_{3 D}(\underline{\gamma}, \underline{\underline{\chi}})=2 \sigma_{0} \int_{0}^{t / 2} \sqrt{z^{2} \boldsymbol{\chi}^{T} \mathbf{Q}_{b} \boldsymbol{\chi}+\boldsymbol{\gamma}^{T} \mathbf{Q}_{s} \boldsymbol{\gamma}} d z \approx \frac{1}{2} \sum_{g=1}^{n g} M_{0} \omega_{g} \sqrt{\left(1+\xi_{g}\right)^{2} \boldsymbol{\chi}^{T} \mathbf{Q}_{b} \boldsymbol{\chi}+\frac{16}{t^{2}} \boldsymbol{\gamma}^{T} \mathbf{Q}_{s} \boldsymbol{\gamma}}$

\footnotetext{
${ }^{1}$ The notation $\pi_{3 D}$ is adopted to keep in mind that it is obtained as the result of an upscaling procedure from a $3 \mathrm{D}$ continuum.
} 
where $\xi=4 z / t-1$ and $\omega_{g}$ is the weighting factor of the Gauss point $\xi_{g}$. It is now quite easy to see that, in the case of a one-point integration rule $\left(\xi_{1}=0, \omega_{1}=2\right)$, equation (17) reduces to (13) along with (12).

Choosing different values of $n g$ will then lead to different choices of the working strength criterion, which will tend to the one obtained with (16) when $n g$ increases. It can be observed that, even with $n g=1$ (i.e. (13)), a very good approximation is obtained, differing by a few percents only from (16). In the following, we will continue working with expression (17) for its generality, although the numerical example will be performed with $n g=1$ so that now :

$$
\pi(\underline{\gamma}, \underline{\underline{\chi}})=\frac{1}{2} \sum_{g=1}^{n g} M_{0} \omega_{g} \sqrt{\left(1+\xi_{g}\right)^{2} \chi^{T} \mathbf{Q}_{b} \chi+\frac{16}{t^{2}} \gamma^{T} \mathbf{Q}_{s} \gamma}
$$

In our point of view, upper bounds will be obtained with respect to a criterion described by a fixed value of $n g$. In particular, computations with a given $n g$ will not produce upper bounds with respect to the criterion obtained with (16). However, post-processing the optimal collapse mechanism to compute the maximal resisting work with expression (16) would still be possible. This has not been done so that upper bounds with $n g=1$ can be compared with the static lower bounds obtained in [22] with criterion (11).

From (18), one has :

$$
\Pi(\underline{n} ; \llbracket w \rrbracket, \llbracket \underline{\beta} \rrbracket)=\frac{1}{2} \sum_{g=1}^{n g} M_{0} \omega_{g} \sqrt{\left(1+\xi_{g}\right)^{2} \llbracket \boldsymbol{\beta} \rrbracket^{T} \mathbf{Q}_{n} \llbracket \boldsymbol{\beta} \rrbracket+\frac{16}{3 t^{2}} \llbracket w \rrbracket^{2}}
$$

Finally, since $\mathbf{Q}_{b}, \mathbf{Q}_{s}$ and $\mathbf{Q}_{n}$ are positive definite matrices, both expressions (18) and (19) can be rewritten in the form of a sum of norms, namely :

$$
\begin{gathered}
\pi(\underline{\gamma}, \underline{\underline{\chi}})=\frac{1}{2} \sum_{g=1}^{n g} M_{0} \omega_{g} \sqrt{\left\|\mathbf{C}_{b g}^{T} \boldsymbol{\chi}\right\|^{2}+\left\|\mathbf{C}_{s}^{T} \boldsymbol{\gamma}\right\|^{2}} \\
\Pi(\underline{n} ; \llbracket w \rrbracket, \llbracket \underline{\beta} \rrbracket)=\frac{1}{2} \sum_{g=1}^{n g} M_{0} \omega_{g} \sqrt{\left\|\mathbf{C}_{n g}^{T} \llbracket \boldsymbol{\beta} \rrbracket\right\|^{2}+\frac{16}{3 t^{2}} \llbracket w \rrbracket^{2}}
\end{gathered}
$$

where the so-called Cholesky matrices $\mathbf{C}_{b g}, \mathbf{C}_{s}$ and $\mathbf{C}_{n g}$ are defined as :

$$
\mathbf{C}_{b g}=\frac{1+\xi_{g}}{\sqrt{3}}\left[\begin{array}{ccc}
2 & 0 & 0 \\
1 & \sqrt{3} & 0 \\
0 & 0 & 1
\end{array}\right] ; \mathbf{C}_{s}=\frac{4}{\sqrt{3} t}\left[\begin{array}{ll}
1 & 0 \\
0 & 1
\end{array}\right] ; \mathbf{C}_{n g}=\frac{1+\xi_{g}}{\sqrt{3}}\left[\begin{array}{ll}
2 & 0 \\
0 & 1
\end{array}\right]
$$

It is worth noting from the examination of equations (20) and (21) that, in the thin plate limit, that is as the plate thickness $t$ tends to zero, the terms associated either with the shear strain $\underline{\gamma}$ or the transversal velocity jump $\llbracket w \rrbracket$ are going to infinity. Therefore, this asymptotic case can only be treated by means of virtual kinematic fields satisfying the Love-Kirchhoff 
conditions : $\underline{\gamma}=\underline{\nabla w}-\underline{\beta}=\underline{0}$ and $\llbracket w \rrbracket=0$ which imply $\underline{\underline{\chi}}=\underline{\underline{\nabla^{s} \nabla w}}$ and $\llbracket \underline{\beta} \rrbracket=\llbracket \frac{\partial w}{\partial n} \rrbracket \underline{n}$. Thus, computing for instance the support function (21), it comes out

$$
\Pi\left(\underline{n} ; 0, \llbracket \frac{\partial w}{\partial n} \rrbracket \underline{n}\right)=\left|\llbracket \frac{\partial w}{\partial n} \rrbracket\right| \sigma_{0} \frac{2}{\sqrt{3}} \int_{-t / 2}^{t / 2}|z| d z=\frac{2}{\sqrt{3}} M_{0}\left|\llbracket \frac{\partial w}{\partial n} \rrbracket\right|
$$

recovering the classical expression of the support function associated with a yield line for a von Mises plate in pure bending.

\section{Finite element discretization}

In this section, the domain $S$ of the plate is supposed to be discretized by $N_{E}$ threenoded triangular finite elements. The important feature of the considered elements is that the degrees of freedom are attached to the element and not to a geometrical node shared by several adjacent elements as is usually the case. Two different interpolations will be investigated : the first one (w3) assumes a linear interpolation of both $w$ and $\beta$ whereas the second one $(w 6)$ assumes a quadratic interpolation for $w$ and a linear interpolation for $\beta$. In both cases, the degrees of freedom attached to an element $e$ will be noted $\mathbf{u}_{e}$ and are arranged as follows :

- for $w 3$ :

$$
\mathbf{u}_{e}=\left\langle\begin{array}{lllllll}
w^{1} & w^{2} & w^{3} & \beta_{x}^{1} & \beta_{y}^{1} & \ldots & \beta_{y}^{3}
\end{array}\right\rangle^{T}
$$

- for $w 6$ :

$$
\mathbf{u}_{e}=\left\langle\begin{array}{lllllll}
w^{1} & \ldots & w^{6} & \beta_{x}^{1} & \beta_{y}^{1} & \ldots & \beta_{y}^{3}
\end{array}\right\rangle^{T}
$$

where $x^{i}$ denotes the value of quantity $x$ at the node $i$ inside the element. Here, the total number of degrees of freedom will thus be $9 N_{E}$ for $w 3$ and $12 N_{E}$ for $w 6$ and it is important to keep in mind that, at a given geometrical node, there will be as many different values of $\mathbf{u}$ as the number of elements sharing this node.

\subsection{Contribution of the first term associated with curvature and shear strains}

The curvatures and shear strains are expressed at the three corners of the element by :

$$
\chi_{e}^{i}=\mathbf{B}_{i}^{b} \mathbf{u}_{e} \quad ; \quad \gamma_{e}^{i}=\mathbf{B}_{i}^{s} \mathbf{u}_{e} \quad \forall i=1, \ldots, 3
$$

It is to be noted that $\chi$ is in fact constant within the element whereas $\gamma$ is linear for both interpolations. Therefore, the strain variables vary at most linearly inside the element. The integrand in $P_{r m}^{\text {strain }}$ being a convex function of the strain variables which vary like a simplex in a triangle with straight edges, the elementary integral in $P_{r m}^{\text {strain }}$ can therefore be 
approximated by excess using the values at the three vertices following the same method as the one employed in [27] for a different criterion :

$$
P_{r m}^{\text {strain }}\left(w, \beta_{x}, \beta_{y}\right) \lesssim \sum_{e=1}^{N_{E}} \sum_{i=1}^{3} \sum_{g=1}^{n g} M_{0} \frac{A_{e}}{3} \frac{\omega_{g}}{2} \sqrt{\left\|\mathbf{C}_{b g}^{T} \mathbf{B}_{i}^{b} \mathbf{u}_{e}\right\|^{2}+\left\|\mathbf{C}_{s}^{T} \mathbf{B}_{i}^{s} \mathbf{u}_{e}\right\|^{2}}
$$

where $A_{e}$ is the area of element $e$.

Introducing, for $p=1, \ldots, 3 N_{E}$, the auxiliary variable $\boldsymbol{\rho}^{g p}$ defined as :

$$
\boldsymbol{\rho}_{1 \rightarrow 3}^{g p}=\left[\begin{array}{c}
\boldsymbol{\rho}_{1}^{g p} \\
\boldsymbol{\rho}_{2}^{g p} \\
\boldsymbol{\rho}_{3}^{g p}
\end{array}\right]=\mathbf{C}_{b g}^{T} \mathbf{B}_{i}^{b} \mathbf{u}_{e} \quad ; \quad \boldsymbol{\rho}_{4 \rightarrow 5}^{g p}=\left[\begin{array}{c}
\boldsymbol{\rho}_{4}^{g p} \\
\boldsymbol{\rho}_{5}^{g p}
\end{array}\right]=\mathbf{C}_{s}^{T} \mathbf{B}_{i}^{s} \mathbf{u}_{p}
$$

equation $(22)$ now reads as :

$$
P_{r m}^{\text {strain }}\left(w, \beta_{x}, \beta_{y}\right) \lesssim M_{0} \sum_{p=1}^{3 N_{E}} \frac{A_{e}}{3} \sum_{g=1}^{n g} \frac{\omega_{g}}{2}\left\|\boldsymbol{\rho}^{g p}\right\|
$$

\subsection{Contribution of the second discontinuity term}

Let us consider a given edge $j$ in the finite element mesh. If $j$ is not on a boundary, then there exists two elements $e$ and $e^{\prime}$ sharing the edge $j$. The discontinuities are computed at both nodes of the edge (denoted respectively by $i_{1}$ (resp. $i_{2}$ ) in element $e$ and $i_{1}^{\prime}$ (resp. $i_{2}^{\prime}$ ) in element $e^{\prime}$ ) plus the mid-side node for the discontinuity of $w$ for the $w 6$ interpolation. The discontinuities are collected in the following vector :

- for $w 3$ :

$$
\llbracket \mathbf{u} \rrbracket^{j}=\left\langle\llbracket w \rrbracket^{1} \quad \llbracket w \rrbracket^{2} \quad \llbracket \beta_{n} \rrbracket^{1} \quad \llbracket \beta_{t} \rrbracket^{1} \quad \llbracket \beta_{n} \rrbracket^{2} \quad \llbracket \beta_{t} \rrbracket^{2}\right\rangle^{T}
$$

- for $w 6$ :

$$
\llbracket \mathbf{u} \rrbracket^{j}=\left\langle\llbracket w \rrbracket^{1} \quad \llbracket w \rrbracket^{2} \quad \llbracket w \rrbracket^{3} \quad \llbracket \beta_{n} \rrbracket^{1} \quad \llbracket \beta_{t} \rrbracket^{1} \quad \llbracket \beta_{n} \rrbracket^{2} \quad \llbracket \beta_{t} \rrbracket^{2}\right\rangle^{T}
$$

where $\llbracket x \rrbracket^{1}=x_{e}^{i_{1}}-x_{e^{\prime}}^{i_{1}^{\prime}}, \llbracket x \rrbracket^{2}=x_{e}^{i_{2}}-x_{e^{\prime}}^{i_{2}^{\prime}}$ and $\llbracket x \rrbracket^{3}=x_{e}^{i_{3}}-x_{e^{\prime}}^{i_{3}^{\prime}}$ if $i_{3}, i_{3}^{\prime}$ represent the mid-side nodes of the edge. The discontinuities of the rotation are expressed in terms of normal and tangential components along the edge. The linear relation between $\llbracket \mathbf{u} \rrbracket^{j}$ and the nodal values $\mathbf{u}_{j}$ along the edge is written as $\llbracket \mathbf{u} \rrbracket^{j}=\mathbf{D}_{j} \mathbf{u}_{j}$.

We also introduce the following auxiliary variables defined as :

- for $w 3$ :

$$
\left[\begin{array}{c}
\boldsymbol{\mu}^{j g 1} \\
\boldsymbol{\mu}^{j g 2}
\end{array}\right]=\left[\begin{array}{cccc}
\frac{4}{\sqrt{3} t} & 0 & \mathbf{0} & \mathbf{0} \\
0 & 0 & \mathbf{C}_{n g}^{T} & \mathbf{0} \\
0 & \frac{4}{\sqrt{3} t} & \mathbf{0} & \mathbf{0} \\
0 & 0 & \mathbf{0} & \mathbf{C}_{n g}^{T}
\end{array}\right] \mathbf{D}_{j} \mathbf{u}^{j}
$$


- for $w 6$ :

$$
\left[\begin{array}{c}
\boldsymbol{\mu}^{j g 1} \\
\boldsymbol{\mu}^{j g 2} \\
\boldsymbol{\mu}^{j g 3}
\end{array}\right]=\left[\begin{array}{ccccc}
\frac{4}{\sqrt{3} t} & 0 & 0 & \mathbf{0} & \mathbf{0} \\
0 & 0 & 0 & \mathbf{C}_{n g}^{T} & \mathbf{0} \\
0 & \frac{4}{\sqrt{3} t} & 0 & \mathbf{0} & \mathbf{0} \\
0 & 0 & 0 & \mathbf{0} & \mathbf{C}_{n g}^{T} \\
0 & 0 & \frac{8}{\sqrt{3} t} & \mathbf{0} & \mathbf{0} \\
0 & 0 & 0 & \mathbf{C}_{n g}^{T} & \mathbf{C}_{n g}^{T}
\end{array}\right] \mathbf{D}_{j} \mathbf{u}^{j}
$$

For $w 3$, the jumps vary linearly along an edge, the second term of the maximum resisting work can therefore be approximated by excess by evaluating the support function at each end node and summing over all active edges :

$$
P_{r m}^{d i s c}\left(w, \beta_{x}, \beta_{y}\right) \lesssim \sum_{j=1}^{N_{D}} \sum_{i=1}^{2} \sum_{g=1}^{n g} M_{0} \frac{l_{j}}{2} \frac{\omega_{g}}{2}\left\|\boldsymbol{\mu}^{j g i}\right\|
$$

where $l_{j}$ is the length of edge $j$ and $N_{D}$ is the number of all active edges.

As regards $w 6$, the jump of $w$ varies quadratically, hence it is not possible to obtain an upper bound approximation. It has been decided to use the same formula as before but on both half of the edge, such that we obtain :

$$
P_{r m}^{d i s c}\left(w, \beta_{x}, \beta_{y}\right) \approx \sum_{j=1}^{N_{D}} \sum_{i=1}^{3} \sum_{g=1}^{n g} M_{0} \frac{l_{j}}{4} \frac{\omega_{g}}{2}\left\|\boldsymbol{\mu}^{j g i}\right\|
$$

\subsection{Boundary conditions}

Active edges correspond to all edges which actually contribute to the maximum resisting work. This includes all internal edges and all boundary edges except those on the free part of the boundary. For other parts of the boundary (e.g. simple supports), we need to modify relations $(25)$ and $(26)$.

Indeed, for fully clamped parts, the relation holds true (since all components of $\llbracket \mathbf{u} \rrbracket$ contribute to the dissipation, but when some components of $\mathbf{u}$ are free (e.g. $\beta_{n}$ for simple supports) the corresponding contribution should be zero. In this case, the corresponding line (denoted by $I$ ) in the definition of $\boldsymbol{\mu}$ should be replaced by $\boldsymbol{\mu}_{I}=0$. For computational simplicity, the components of the angular velocity field $\beta$ are given in terms of normal and tangential components for the boundary conditions. Therefore, the boundary conditions can be enforced directly on the components of $\boldsymbol{\mu}$ as follows :

$$
\begin{array}{ll}
w=0 & \Rightarrow \boldsymbol{\mu}_{2}=\boldsymbol{\mu}_{3}=0 \\
\beta_{n}=0 & \Rightarrow \boldsymbol{\mu}_{1}=\boldsymbol{\mu}_{3}=0 \\
\beta_{t}=0 & \Rightarrow \boldsymbol{\mu}_{1}=\boldsymbol{\mu}_{2}=0 \\
w=\beta_{n}=0 & \Rightarrow \boldsymbol{\mu}_{3}=0 \\
w=\beta_{t}=0 & \Rightarrow \boldsymbol{\mu}_{2}=0 \\
\beta_{n}=\beta_{t}=0 & \Rightarrow \boldsymbol{\mu}_{1}=0
\end{array}
$$




\subsection{Mathematical programming}

Denoting by $\mathbf{f}$ the loading vector such that $P_{(e)}\left(w, \beta_{x}, \beta_{y}\right)=\mathbf{f}^{T} \mathbf{u}$, we can formulate the global optimization problem as follows :

$$
\begin{aligned}
\lambda^{k i n}=\min & \sum_{p=1}^{3 N_{E}} \sum_{g=1}^{n g} c_{g p} t_{g p}+\sum_{p^{\prime}=1}^{q N_{D}} \sum_{g=1}^{n g} c_{g p^{\prime}}^{\prime} s_{g p^{\prime}} \\
\text { s.t. } & \mathbf{f}^{T} \mathbf{u}=1 \\
& \mathbf{C}_{\boldsymbol{\rho}} \mathbf{B u}-\boldsymbol{\rho}=\mathbf{0} \\
& \mathbf{C}_{\boldsymbol{\mu}} \mathbf{D} \mathbf{u}-\boldsymbol{\mu}=\mathbf{0} \\
& \left\|\boldsymbol{\rho}^{g p}\right\| \leq t_{g p} \\
& \left\|\boldsymbol{\mu}^{g p^{\prime}}\right\| \leq s_{g p^{\prime}} \\
& \boldsymbol{\mu}_{I}=0
\end{aligned}
$$

where $c_{g p}=A_{e} M_{0} \omega_{g} / 6, c_{g p^{\prime}}=l_{j} M_{0} \omega_{g} / 4$, constraints (29c) and (29d) correspond to the assembled form of the definitions (23) and (25)-(26), $q=2$ for $w 3$ and $q=3$ for $w 6$ and $B C$ indicates the set of indexes of the global vector $\boldsymbol{\mu}$ corresponding to the boundary conditions as discussed before.

This problem is, therefore, cast as a SOCP problem with linear equality constraints and quadratic cones. There are $3 N_{E} \cdot n g$ cones of dimension 6 (constraints $\left.(29 \mathrm{e})\right)$ and $q N_{D} \cdot n g$ cones of dimension 4 (constraints (29f)).

Finally, it should be noted that, since $P_{r m}^{\text {strain }}$ and $P_{r m}^{\text {disc }}$ are approximated by excess for $w 3$, problem (29) consists in an exact upper bound kinematic approach and, therefore, will yield a strict upper bound to the ultimate load.

As regards $w 6$, a post-processing procedure is needed to compute exactly $P_{r m}^{\text {disc }}$ using the optimal velocity field obtained from the resolution of (29). The so-obtained load factor being then a true upper bound estimate, although the difference is almost always negligible in practice.

\subsection{Comments on the considered finite elements}

Until now, fully discontinuous finite elements have been considered. Obviously, the case of fully continuous elements is obtained as a particular case if all discontinuity variables ( $\boldsymbol{\mu}$ for instance) are forced to be zero by adding a supplementary constraint in (29).

Since fully continuous elements are available without any supplementary difficulties, it will be interesting to compare their performances with respect to fully discontinuous ones (especially in the thin plate limit). Hence, from now on, 4 different types of finite elements will be considered $^{2}$ :

- w3-c : continuous elements with linear interpolation of the transverse velocity $w$

\footnotetext{
${ }^{2}$ Note that in each case, the rotation vector is linearly interpolated within an element but is continuous for the first two element types and discontinuous for the last two types
} 
- $w 6-c$ : continuous elements with quadratic interpolation of the transverse velocity $w$

- w3-d : discontinuous elements with linear interpolation of the transverse velocity $w$

- $w 6-d$ : discontinuous elements with quadratic interpolation of the transverse velocity $w$

\section{Shear-locking effect in thin plate limit}

\subsection{Shear-locking effect}

Shear-locking for thick plate finite elements can be defined, from an engineering point of view, as the lack of robustness of the considered interpolation in the thin plate limit. From a numerical point of view, this lack of robustness corresponds to a convergence rate with respect to the mesh size $h$ which is not uniform with respect to the plate thickness $t$. For example, let $u_{h, t}$ correspond to the discrete solution of a problem with a mesh size $h$ and a plate thickness $t$ and $u_{0}$ be the exact solution of the corresponding thin plate problem with a vanishing thickness. If the following estimate holds true :

$$
\left\|u_{h, t}-u_{0}\right\| \leq C \frac{h}{t}
$$

where $C$ is a constant which is independent on $h$ and $t$, then shear-locking will occur. Indeed, in order to attain a fixed error $\epsilon$, the mesh size $h$ must behave like $\epsilon t$. Another consequence is that for a fixed mesh size, the quality of the discrete solution will strongly deteriorates (in $1 / t$ ) with smaller thicknesses. An interpretation of the source of the shear-locking phenomenon is that the functional space corresponding to the continuous solution with a nonzero thickness $\mathcal{W}_{t}$ and the functional space of the thin plate solution (zero thickness) $\mathcal{W}_{0}$ are different ${ }^{3}$. If $\mathcal{W}_{h, t}$ is the discrete approximation of $\mathcal{W}_{t}$, then we may have $\mathcal{W}_{h, t} \rightarrow \mathcal{W}_{t}$ when $h \rightarrow 0$ for a fixed $t$ but it is possible that $\mathcal{W}_{h, t} \cap \mathcal{W}_{0}=\emptyset$ so that $\mathcal{W}_{h, t} \cap \mathcal{W}_{0} \nrightarrow \mathcal{W}_{0}$.

This so-called "shear-locking effect" has been widely discussed for elastic plate problems $[28,29]$. Various numerical strategies have been proposed to circumvent it like, for instance, reduced integration, mixed approaches, assumed strains, etc... However, shear-locking for limit analysis plate problems has been less studied. Of the few available papers on this subject $[18,26,30]$, a natural idea was to apply the same stabilizing techniques which proved to be efficient for elastic problems. One major drawback with respect to the kinematic approach is that strain-displacement compatibility equations are often relaxed in some sense and the upper bound status of the solution is lost. Besides, functional spaces involved in upper bound kinematic approaches are larger than those involved in elasticity problems, notably authorizing an admissible class of discontinuities of the virtual velocity fields. For this reason, it seems reasonable to investigate this problem while keeping this difference in mind.

\footnotetext{
${ }^{3}$ The normalization of the power of external loads is assumed to be included in the definition of all functional spaces
} 


\subsection{Discontinuous elements are locking-free}

The purpose of this subsection is not aimed at giving a formal mathematical demonstration but only at providing simple arguments which will then be illustrated by numerical examples in the next section.

Continuous elements with linear interpolation (w3-c) for thick plate yield design have already been considered by some authors [26, 30] and have proved to exhibit shear-locking in the thin plate limit. This is attributed to the fact that, since Love-Kirchhoff kinematics are obtained in the thin plate limit, the kinematic constraint $\underline{\beta}=\underline{\nabla w}$ must hold true inside the element. Since $w$ is linear, the rotation vector must be constant inside an element. Since it is also continuous, it must be constant over the whole domain. Hence, it is easy to understand that, except for trivial cases, the only admissible solution with constant rotation is the null field which cannot satisfy the normalization constraint, so that $\mathcal{W}_{h, t} \cap \mathcal{W}_{0}=\emptyset$ here.

Concerning $w 6$-c, it reduces, in the thin plate limit, to an element with a quadratic transverse velocity interpolation which must be continuous in the whole plate and the gradient of which (the rotation vector $\beta$ due to the Love-Kirchhoff condition) must also be continuous in the whole plate (since $\beta$ is continuous for $w 6-c$ ). However, as is well known, it is impossible to achieve a continuity of both a scalar field and its gradient ( $C^{1}$-continuity) with a quadratic interpolation only. The degree of this interpolation is indeed too weak and leads to trivial fields to be exactly satisfied (e.g. an affine field for the transverse displacement and a uniform gradient). Hence, except in trivial cases where these fields correspond to the solution, the fields obtained by $w 6$-c in the limit will not be able to satisfy the boundary conditions and the normalization of the external load work so that $\mathcal{W}_{h, t} \cap \mathcal{W}_{0}=\emptyset$.

As regards discontinuous elements, the transverse velocity must be continuous in the thin plate limit. Due to the Love-Kirchhoff relation $\beta=\underline{\nabla w}$, the tangential component of the rotation vector must also be continuous by virtue of Hadamard's compatibility condition [20]. Therefore, $w 3$-d reduces to a finite element with piecewise constant rotation element (curvature strains are null) with possible discontinuities of the normal rotation through element edges. This corresponds exactly to a pure yield line (YL) element, so that $\mathcal{W}_{h, t} \cap \mathcal{W}_{0}=\mathcal{W}_{h, Y L}$ here. It is known that yield line elements provide upper bound estimates but cannot, in general, converge to the true limit load even with finer meshes [31]. Hence, it is expected that w3-d will not lock in the thin plate limit but will converge to the corresponding yield line upper bound estimate.

Similarly, w6-d reduces to a piecewise linear rotation element with possible discontinuities of the normal rotation and with constant curvature strains. Contrary to what was obtained in [10], this element actually converges to the exact thin plate limit load when refining the mesh, confirming the results of [32]. The results presented in [10] have indeed been improved by fixing an error present in the initial numerical code which yielded a very poor convergence rate. Moreover, theoretical $\Gamma$-convergence results for this quadratic thin plate element are currently investigated. Therefore, since the functional space spanned by 
the $w 6$-d element contains the functional space spanned by the thin plate (T6) element $\left(\mathcal{W}_{h, t} \cap \mathcal{W}_{0}=\mathcal{W}_{h, T 6}\right)$, it is also expected that $w 6$-d will not lock in the thin plate limit and will converge to the corresponding upper bound estimate obtained with a $\mathrm{T} 6$ thin plate element.

Finally, let us point out that, in the following, an element will be said to lock if and only if $\mathcal{W}_{h, t} \cap \mathcal{W}_{0}=\emptyset$. The case when $\mathcal{V}_{h}=\mathcal{W}_{h, t} \cap \mathcal{W}_{0} \neq \emptyset$ will correspond to a locking-free element. However, it is not ensured that $\mathcal{V}_{h} \rightarrow \mathcal{W}_{0}$ when $h \rightarrow 0$ e.g. we have $\mathcal{V}_{h}=\mathcal{W}_{h, T 6} \rightarrow \mathcal{W}_{0}$ for $w 6$-d but $\mathcal{V}_{h}=\mathcal{W}_{h, Y L} \nrightarrow \rightarrow \mathcal{W}_{0}$ for $w 3$-d. But, in each case a finite upper bound estimate will be obtained in the thin plate limit.

\subsection{A simple test to detect shear-locking}

In this subsection, a simple test is proposed to detect a potential shear-locking phenomenon. The idea is to solve a mathematical programming inspired from (29) to check if $\mathcal{W}_{h, t} \cap \mathcal{W}_{0}=\emptyset$ or not. To this purpose, the following problem is defined :

$$
\begin{aligned}
& \lambda_{0}=\min \sum_{p=1}^{3 N_{E}} \sum_{g=1}^{n g} c_{g p} t_{g p}+\sum_{p^{\prime}=1}^{q N_{D}} \sum_{g=1}^{n g} c_{g p^{\prime}}^{\prime} s_{g p^{\prime}} \\
& \text { s.t. } \mathbf{f}^{T} \mathbf{u}=1 \\
& \mathrm{C}_{\rho} \mathrm{Bu}-\rho=0 \\
& \mathrm{C}_{\boldsymbol{\mu}} \mathrm{Du}-\boldsymbol{\mu}=\mathbf{0} \\
& \left\|\boldsymbol{\rho}_{1 \rightarrow 3}^{g p}\right\| \leq t_{g p} \\
& \forall p=1, \ldots, 3 N_{E} \\
& \boldsymbol{\rho}_{4 \rightarrow 5}^{g p}=\mathbf{0} \\
& \left\|\boldsymbol{\mu}_{2 \rightarrow 3}^{g p^{\prime}}\right\| \leq s_{g p^{\prime}} \\
& \boldsymbol{\mu}_{1}^{g p^{\prime}}=0 \\
& \boldsymbol{\mu}_{I}=0
\end{aligned}
$$

The structure of this problem is exactly the same as (29) but all auxiliary variables associated to shear strains and discontinuities have been enforced to zero, which implicitly corresponds to the enforcement of the Love-Kirchhoff kinematics since $\boldsymbol{\rho}_{4 \rightarrow 5}^{g p}=\mathbf{0}$ is equivalent to $\boldsymbol{\gamma}_{e}^{i}=0$ and $\boldsymbol{\mu}_{1}^{g p^{\prime}}=0$ is equivalent to $\llbracket w \rrbracket^{j i}=0$. Obviously, the formulation could be simplified but it has been kept as such to illustrate the fact that it corresponds to the limit when $t \rightarrow 0$ of problem (29). In particular, it is to be noted that the solution does not depend on $t$ anymore.

The resolution of (30) is then a direct information about the shear-locking behavior of the considered finite element interpolation. Indeed, two cases may arise :

- (30) is feasible, then $\lambda_{0}<\infty$ and corresponds to the limit when $t \rightarrow 0$ of the solution of $(29)$

- (30) is infeasible, then $\mathcal{W}_{h, t} \cap \mathcal{W}_{0}=\emptyset$ and the element will lock 

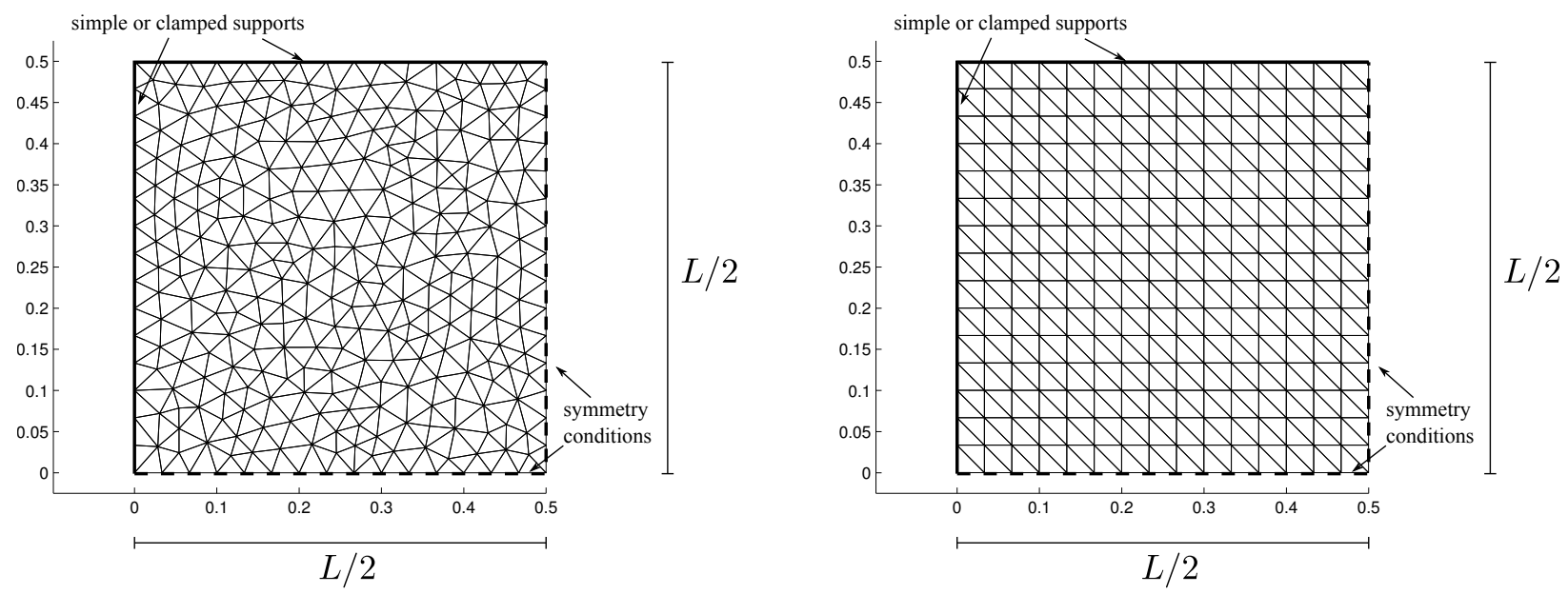

Figure 2: Geometry and meshes (left: unstructured, right: structured) of the square plate problem

\begin{tabular}{c|cccc|c}
\hline Mesh & $w 3-c$ & $w 6-c$ & $w 3-d$ & $w 6-d$ & $\mathrm{YL}$ \\
\hline structured & INFEAS. & INFEAS. & 27.71 & 25.46 & 27.71 \\
\hline unstructured & INFEAS. & INFEAS. & 33.03 & 25.64 & 33.03 \\
\hline
\end{tabular}

Table 1: Shear-locking test results for the simply supported square plate. INFEAS. means that MosEK returned a primal infeasibility status, otherwise non-dimensional limit load values $\lambda=p L^{2} / M_{0}$ are given.

The interest of this simple test is that a single feasibility check is necessary to detect shearlocking, in particular there is no need to perform computations with increasingly smaller values of the thickness. Obviously, it also provides the corresponding thin plate upper bound estimates for locking-free elements.

\section{Numerical results}

In this section, illustrative applications are considered to investigate the performance of the proposed elements. The finite element meshes have been generated using GmSH [33] and the second-order cone programs have been solved using the MOSEK conic solver [34]. In all examples, only one integration point has been used in the half-thickness.

\subsection{Shear-locking tests on the square plate problem}

The classical benchmark problem of a square plate of size $L \times L$ under uniform loading with simple and clamped supports is considered. To illustrate the conclusions of section 5 , the test (30) is solved for the 4 finite elements considered in this work. A structured and an unstructured mesh of the upper-left quarter of the plate with 15 elements per half-side have been used for the computations (Figure 2). As a comparison, upper bound estimates using pure yield line (YL) elements have also been computed on the same meshes.

Shear-locking test results for the square plate example have been reported in Table 1 for simple supports and in Table 2 for clamped supports. For all computations, it can 


\begin{tabular}{c|cccc|c}
\hline Mesh & $w 3-c$ & $w 6-c$ & $w 3-d$ & $w 6-d$ & YL \\
\hline structured & INFEAS. & INFEAS. & 55.43 & 45.19 & 55.43 \\
\hline unstructured & INFEAS. & INFEAS. & 56.28 & 45.23 & 56.28 \\
\hline
\end{tabular}

Table 2: Shear-locking test results for the clamped square plate. INFEAS. means that MosEK returned a primal infeasibility status, otherwise non-dimensional limit load values $\lambda=p L^{2} / M_{0}$ are given.

be observed that the previous remarks concerning the locking phenomenon for continuous elements $w 3-c$ and $w 6-c$ are verified, since all problems are infeasible, meaning that both continuous elements lock in the thin plate limit. On the contrary, as expected both discontinuous elements $w 3-\mathrm{d}$ and $w 6$-d pass the shear-locking tests for all problems. It can also be observed that the thin plate limit load obtained with the $w 3$-d element are the same as those obtained using a pure yield line thin plate element as expected.

\subsection{Square plates: influence of the slenderness ratio}

Next, the influence of the slenderness ratio defined as $L / t$ on the collapse multiplier has been studied. The square plate problem has been solved using three unstructured uniform meshes with $N=3$ (coarse mesh) or $N=15$ (fine mesh) elements per half-side. Collapse multipliers against slenderness ratios are shown in Figures 3 for the different elements and both meshes. The black curve corresponds to the lower bound estimates obtained from a static approach using newly developed equilibrium elements for thick plates [22].

First, it can be observed that all elements produce roughly the same values for small values of the slenderness ratio (shear-dominated failure). Collapse multipliers obtained here for clamped and simply supported plates indicate that perfect shear solution is independent of the edge boundary conditions, as concluded in [18].

It can be seen that as the slenderness ratio increases, the solutions obtained using $w 3-\mathrm{d}$ and w6-d converge to a constant value which correspond, at the limit, to the Kirchhoff (thin plates) values obtained previously i.e. the corresponding yield line estimate for $w 3-\mathrm{d}$ and a value close to the exact thin plate collapse load for $w 6$-d. In particular, it can be noted that, for the whole range of slenderness ratios, the upper bound estimates obtained with $w 6$-d are extremely close to the static lower bound estimates, so that the exact collapse load is bracketed with an extremely good accuracy.

However, as regards continuous elements $w 3-c$ and $w 6-c$, the shear locking effect is clearly observed as the upper bound estimates diverge from the exact value with increasing slenderness ratios. The effect is less pronounced for $w 6$-c than for $w 3-c$ and it is also delayed with finer meshes as expected. One can also notice that the shear locking effect is stronger for clamped boundary conditions.

Figures 4 compares the present collapse load multipliers with those obtained in [18] using a stabilized Discrete Shear Gap (DSG) method. It can be observed that the method presented here provides slightly better solutions than the DSG method for clamped plates, while results obtained for simply supported plates results by both $w 6$-d and DSG elements are in good agreement. 


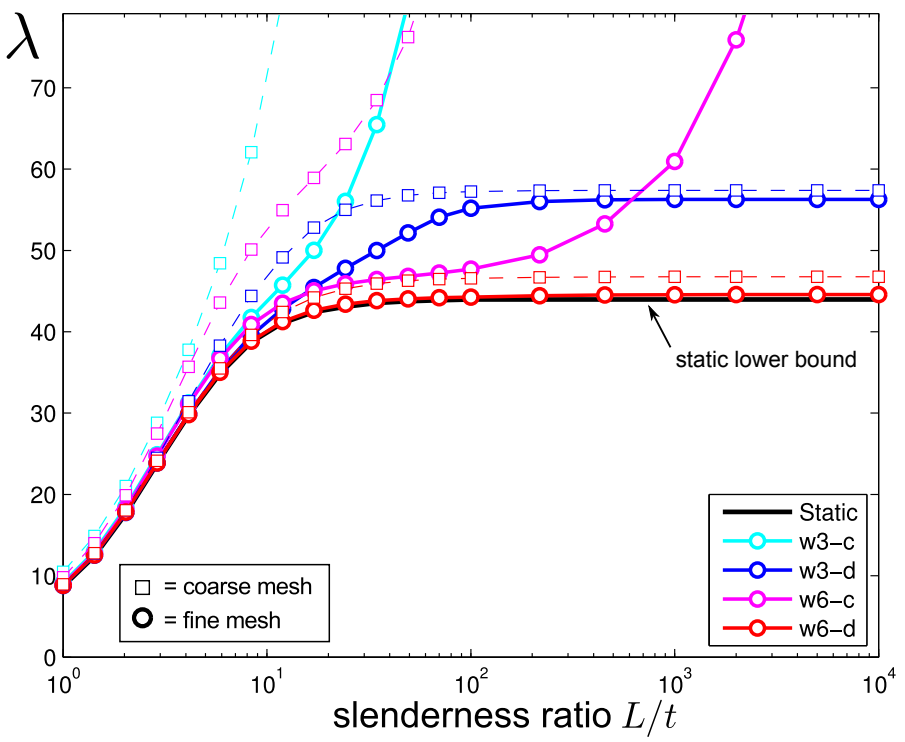

(a) Clamped

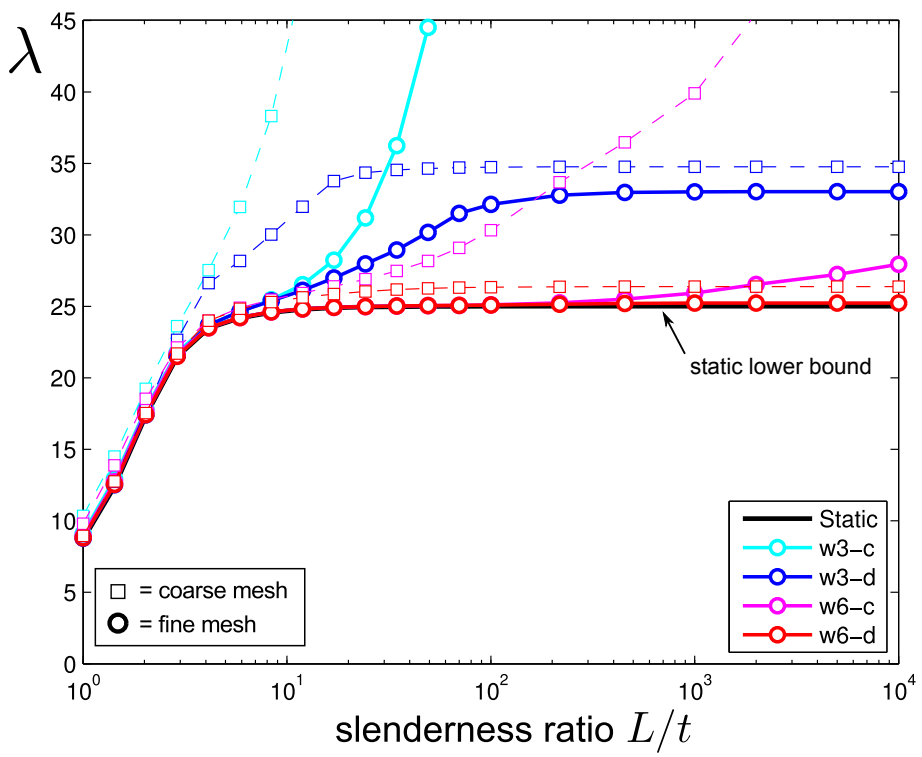

(b) Simply supported

Figure 3: Square plates: collapse load multiplier vs slenderness ratio 


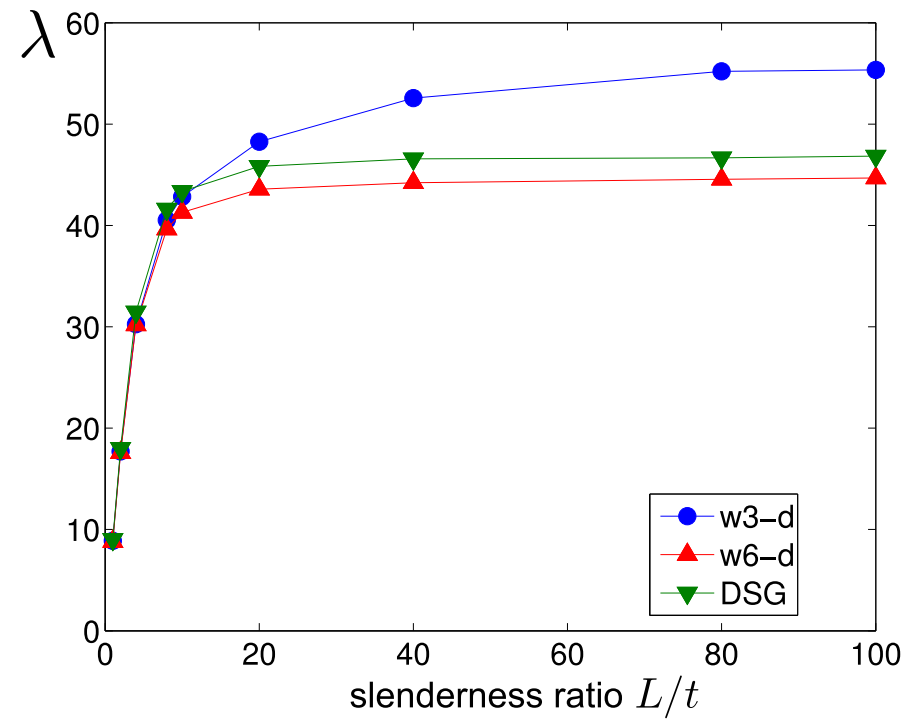

(a) Clamped

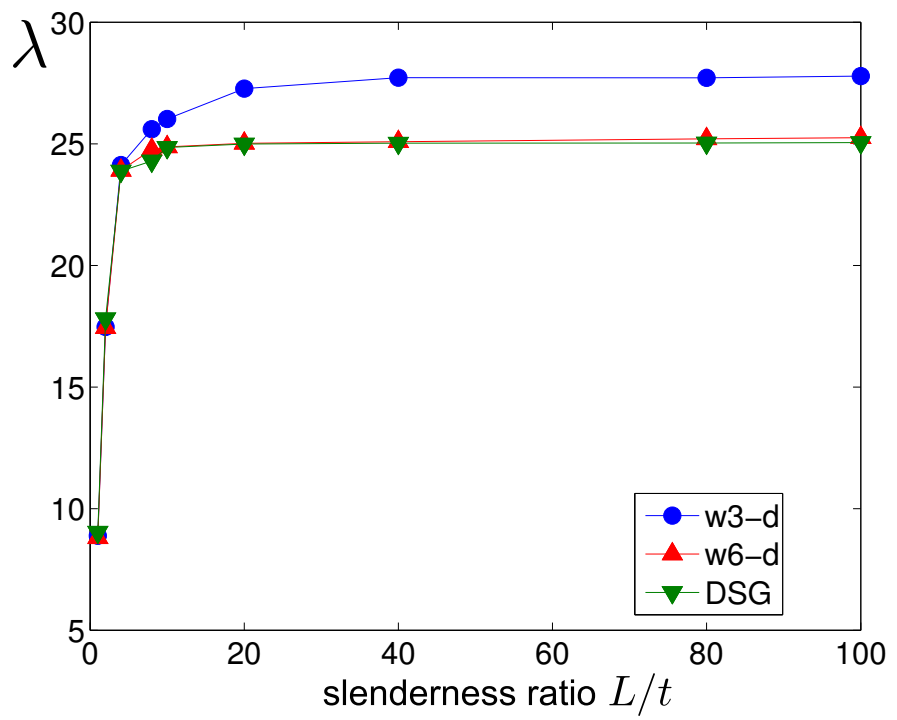

(b) Simply supported

Figure 4: Collapse load multiplier comparison 


\subsection{Circular plates}

The next example treats the classical problem of simply supported and built-in circular plates subjected to a uniformly distributed pressure $p$. Here numerical solutions have been obtained by solving a quarter of the plate, Figure 5 where $R=1 \mathrm{~m}$. Non-dimensional collapse multipliers $\lambda=p R^{2} / M_{0}$ for various slenderness ratios are given in Table 3 . It can be observed that the solutions obtained using $w 6$-d elements are close to those reported in [30] as well as to the lower bound estimates of [22].

In particular, the perfect shear solution $p R^{2} / M_{0}=4 / \sqrt{3}(2 R / t) \approx 2.31(2 R / t)$ is almost exactly computed, the exact failure mechanism corresponding to a uniform transversal velocity and zero rotations, which can be reproduced using discontinuous elements whereas the elements used in [30] cannot. Similarly, the thin plate solution for the clamped problems involves rotation discontinuities along the curved edge so that the performance of $w 6-\mathrm{d}$ is better than the solution of [30]. Finally, in the simply supported case, the upper bound estimates with $w 6$-d are slightly higher (by 1\%) than [30] but the exact upper bound status of the latter results cannot be rigorously assured since a relaxation of the strain compatibility equations has been used.

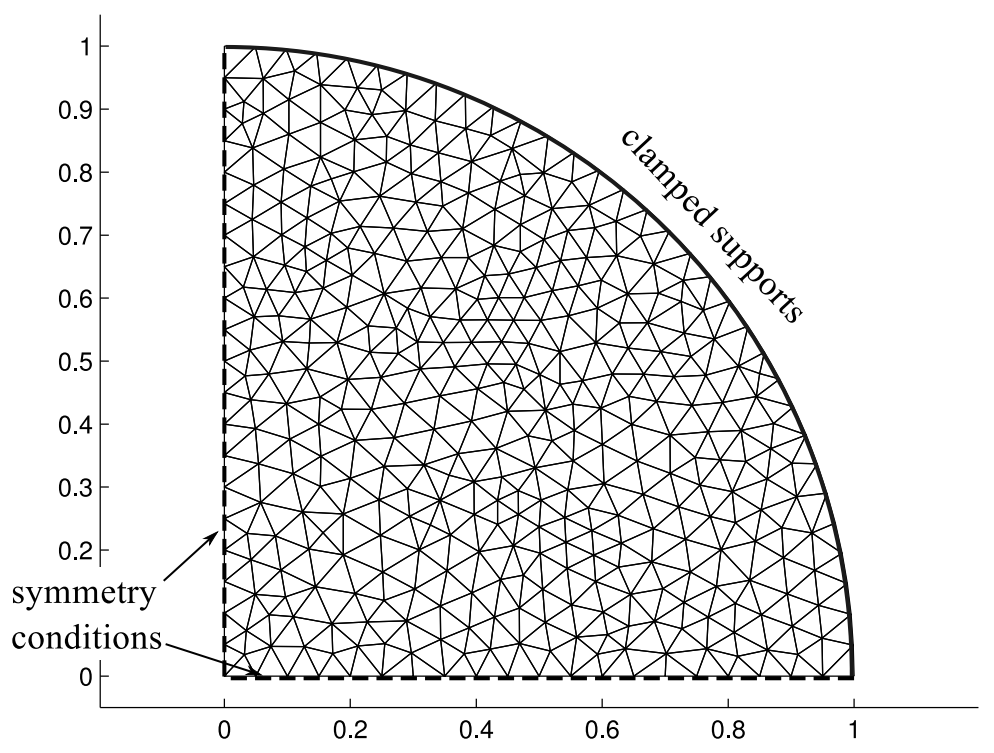

Figure 5: Circular plate: geometry and finite element mesh

\subsection{L-shape plate}

Further illustration of the presented method can be made by examining an L-shaped plate subjected to a uniform load and with geometry and kinematic boundary conditions indicated in Figure 6. An upper bound of $\lambda^{+}=p^{+} L^{2} / M_{0}=\frac{32}{3 \sqrt{3}} \approx 6.16$ can be obtained using the yield line theory with the particular mechanism of a yield line crossing the middle 
Table 3: Results for clamped \& simply supported circular plates subjected to uniform load

\begin{tabular}{cccccccccc}
\hline & \multicolumn{4}{c}{ simply supported } & & \multicolumn{4}{c}{ clamped } \\
\cline { 2 - 4 } \cline { 7 - 9 }$t$ & Static $[22]$ & $w 3-\mathrm{d}$ & $w 6-\mathrm{d}$ & Result in [30] & & Static $[22]$ & $w 3-\mathrm{d}$ & $w 6-\mathrm{d}$ & Result in $[30]$ \\
\hline 1 & 2.309 & 2.310 & 2.310 & 2.370 & & 2.309 & 2.310 & 2.310 & 2.370 \\
2 & 4.619 & 4.620 & 4.620 & 4.747 & & 4.619 & 4.620 & 4.620 & 4.740 \\
4 & 6.002 & 6.070 & 6.031 & 6.164 & & 8.283 & 8.297 & 8.291 & 8.778 \\
8 & 6.382 & 6.518 & 6.416 & 6.449 & & 11.018 & 11.125 & 11.035 & 11.893 \\
10 & 6.431 & 6.615 & 6.463 & 6.479 & & 11.516 & 11.694 & 11.531 & 12.378 \\
20 & 6.498 & 6.921 & 6.531 & 6.518 & & 12.270 & 12.920 & 12.287 & 12.990 \\
40 & 6.515 & 7.198 & 6.556 & 6.527 & & 12.478 & 13.859 & 12.496 & 13.126 \\
100 & 6.521 & 7.362 & 6.585 & 6.530 & & 12.538 & 14.499 & 12.559 & 13.165 \\
\hline
\end{tabular}

of the plate. The problem has been solved using three different structured meshes of 150 , 600 and $2400 w 6$-d elements. Upper bound estimates of the collapse multipliers for various slenderness ratios are reported in Table 4 and Figure 7 and compared against lower bound estimates using a static approach [22]. The exact collapse load can be estimated within a relative accuracy of less than $4 \%$ using the coarsest mesh and by less than $1 \%$ using the finest mesh. It can also be seen that the present results for perfect bending plates are in excellent agreement with the yield line based solution. It is interesting to point out that the solutions obtained using only $600 w 6$-d elements are in very good agreement with those achieved in [18] using adaptive DSG elements.

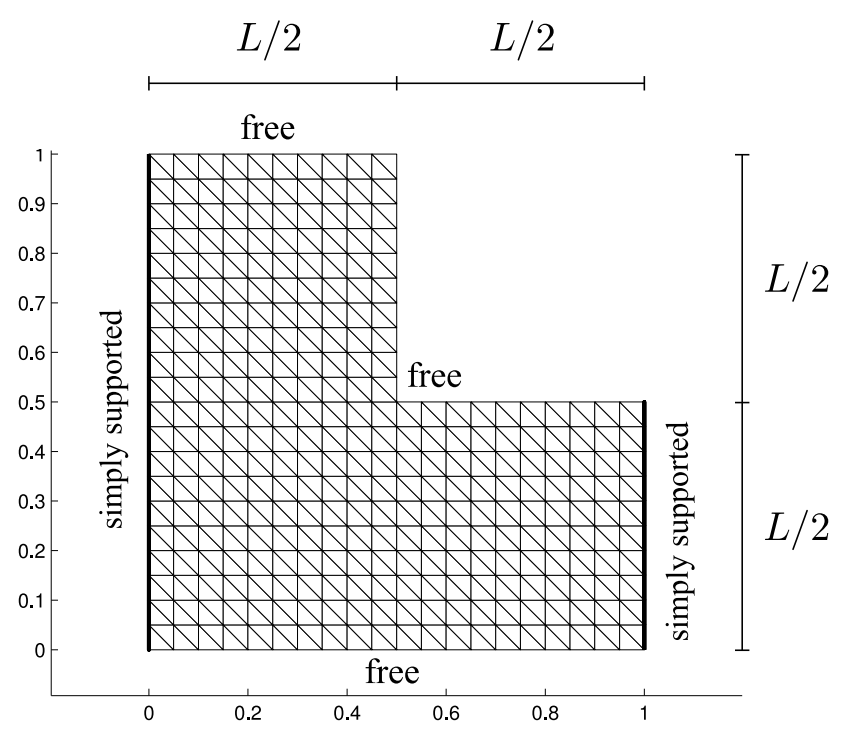

Figure 6: L-shape plate: geometry and finite element mesh

Finally, the differences between the 4 types of elements considered in this work are illustrated by considering the same geometry as before except that a uniformly distributed loading has been applied only to the left half of the plate i.e. : $0 \leq x \leq L / 2,0 \leq y \leq L$ and that the plate is now clamped on both sides. Figures 8-11 represent the optimal transversal 
Table 4: Results for L-shape plates obtained using w6-d

\begin{tabular}{cccccccccc}
\hline nel & $\frac{L}{t}=1$ & 2 & 4 & 8 & 10 & 20 & 40 & 80 & 100 \\
\hline 150 & 3.70 & 5.90 & 6.07 & 6.15 & 6.15 & 6.16 & 6.16 & 6.16 & 6.16 \\
600 & 3.70 & 5.79 & 5.98 & 6.09 & 6.11 & 6.15 & 6.16 & 6.16 & 6.16 \\
2400 & 3.70 & 5.74 & 5.93 & 6.05 & 6.07 & 6.13 & 6.15 & 6.16 & 6.16 \\
Le [18]-adaptive & 3.73 & 5.80 & 5.98 & 6.08 & 6.10 & 6.14 & 6.16 & 6.18 & 6.18 \\
Static [22] & 3.70 & 5.69 & 5.89 & 6.00 & 6.03 & 6.08 & 6.10 & 6.11 & 6.11 \\
\hline \multicolumn{7}{c}{$n e l=$ number of elements } & & &
\end{tabular}

velocity fields obtained with the different elements and for different slenderness ratios. One can clearly observe that the solutions exhibit strong discontinuities in the transverse velocity field which are reproduced by the discontinuous elements. The same comment can be made concerning the rotation field.

\section{Conclusions}

A numerical procedure for performing upper bound yield design of Reissner-Mindlin plates using discontinuous finite elements and second-order cone programming has been presented. The formulation permits discontinuous interpolations of the transverse velocity and rotation fields across all edges shared by adjacent triangles, in addition to shear and bending strains inside the elements. As a result, contrary to continuous elements, shear locking phenomenon can be removed without any additional treatment, and accurate upper bounds on the collapse load factor of Reissner-Mindlin plates can be obtained using a relatively small number of elements. More precisely, as regards the four types of finite elements considered in this paper, the following conclusions can be drawn :

- the linear continuous element (w3-c) yields a strict upper bound but locks very strongly in the thin plate limit, the computed upper bounds diverge from the thin plate solution for high values of the slenderness ratio.

- the quadratic continuous element (w6-c) also yields a strict upper bound and locks in the thin plate limit, although less strongly than (w3-c).

- the linear discontinuous element (w3-d) yields a strict upper bound and does not lock in the thin plate limit. Indeed, although not strictly converging to the thin plate solution for high values of the slenderness ratio, the produced upper bounds stay bounded and the element converges to a pure yield line element which produces sometimes good upper bound estimates of the true thin plate solution.

- the quadratic discontinuous element (w6-d) provides a strict upper bound after applying a post-process computation and does not lock in the thin plate limit. Numerical 


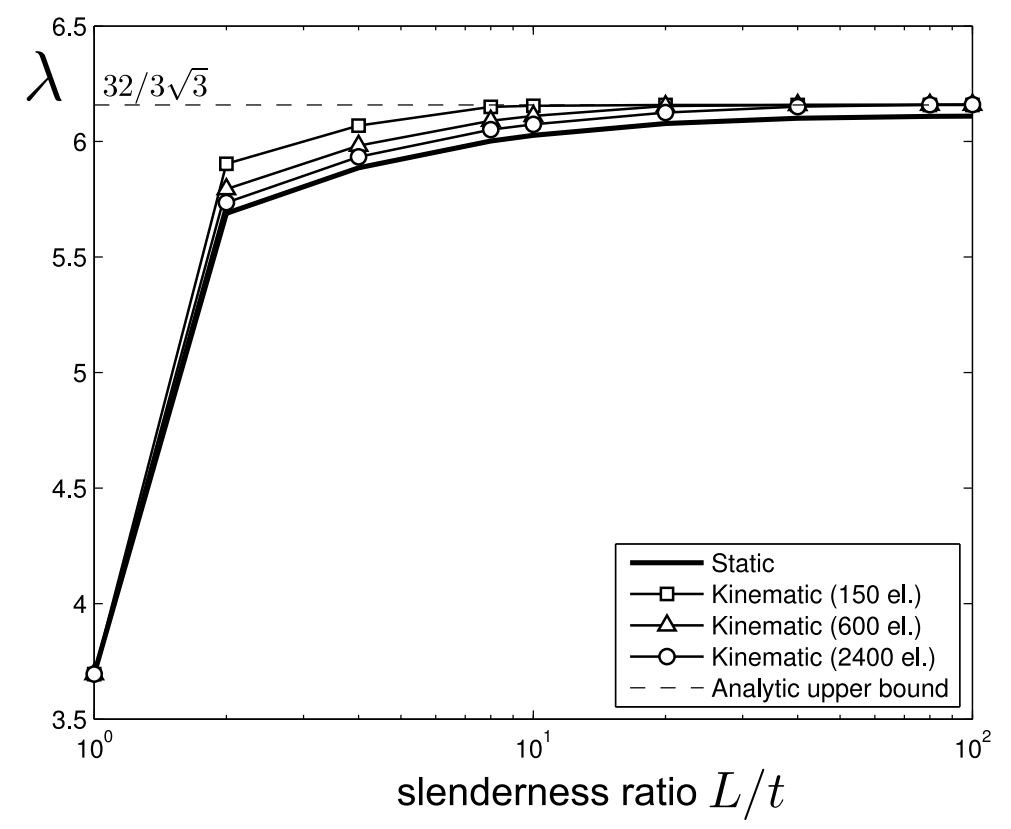

Figure 7: L-shape plate: collapse load multiplier vs slenderness ratio

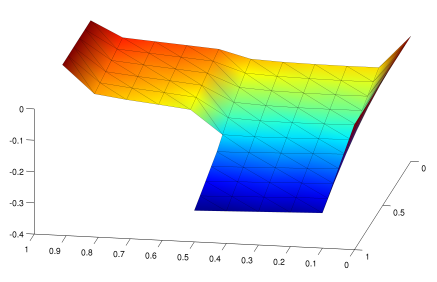

(a) $L / t=1$

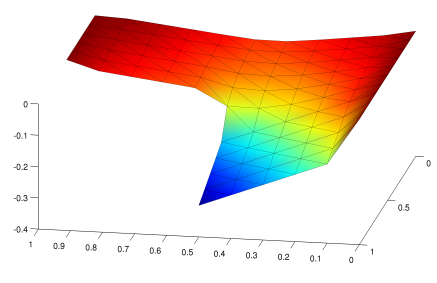

(b) $L / t=4$

Figure 8: w3-c element

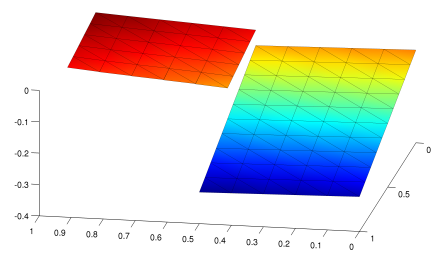

(a) $L / t=1$

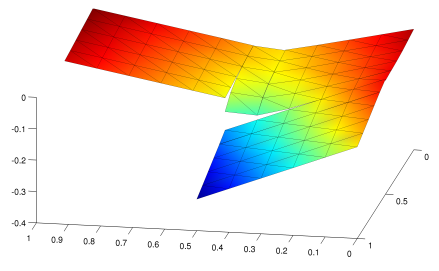

(b) $L / t=4$

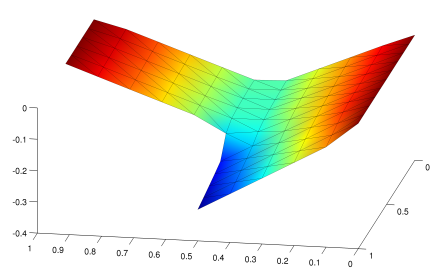

(c) $L / t=10$

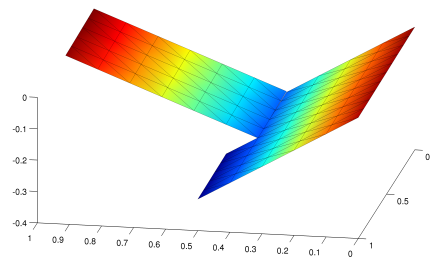

(c) $L / t=10$

Figure 9: w3-d element 


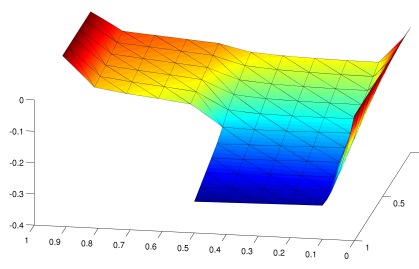

(a) $L / t=1$

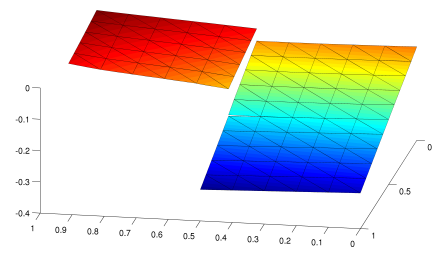

(a) $L / t=1$

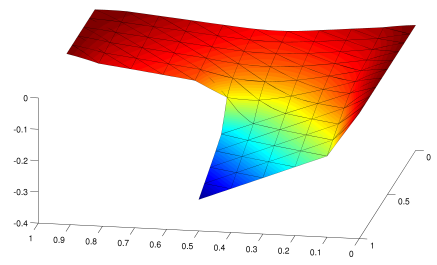

(b) $L / t=4$

Figure 10: w6-c element

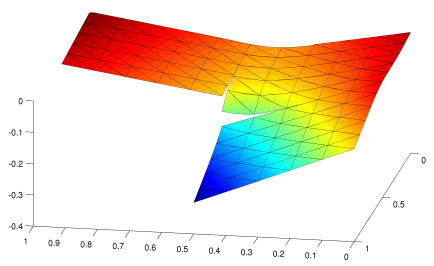

(b) $L / t=4$

Figure 11: w6-d element

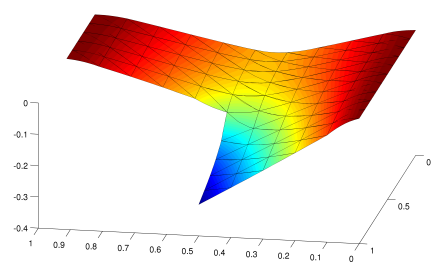

(c) $L / t=10$

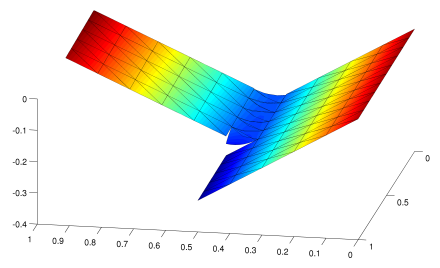

(c) $L / t=10$ 
examples show that it converges to the thin plate solution for high slenderness ratios and gives very good estimates in both shear-dominated and bending-dominated situations when compared to static lower bound estimates.

Obviously, such discontinuous elements will give excellent results in the case when the mesh edges are oriented along the solution discontinuity lines. In general, these directions are not known beforehand but some comments can be made :

- Pure shear solutions will often exhibit transverse velocity discontinuities along the plate boundary or where the loading or material properties change abruptly. Similarly, thin plate solution often exhibit rotation discontinuities on the clamped boundaries. Therefore, one can highly benefit from discontinuous elements at least on these regions, as illustrated by the improvement of bending plate solutions for clamped problems.

- Even if the mesh edges are not oriented along the solution discontinuities, it is compensated by shear or bending strains inside the elements as for traditional continuous elements, reducing at the same time mesh dependency. Therefore, including discontinuities offers an additional optimization variable which can be activated or not in the optimization process.

- As for conforming elements, an adaptive remeshing procedure can still be used to refine the solution in specific regions. One can imagine that such a procedure, using a suitable criterion on the edge direction of the new elements, would be even more efficient for discontinuous elements than for continuous ones.

Finally, the proposed upper bound kinematic approach has been compared to a recently developed lower bound static approach, showing that both bounds are very close, so that the exact collapse load can be bracketed with a very good accuracy. However, both works considered only a constitutive material obeying the von Mises criterion. Further work will therefore investigate other types of strength criterion, more suited to the case of reinforced concrete thick plates for example.

\section{Acknowledgements}

The second author acknowledges the support of the Vietnam National Foundation for Science and Technology Development (NAFOSTED) under grant number 107.02-2013.11

[1] Anderheggen E, Knöpfel H. Finite element limit analysis using linear programming. International Journal of Solids and Structures 1972; 8(12):1413-1431.

[2] Capsoni A, Corradi L. A finite element formulation of the rigid-plastic limit analysis problem. International Journal for Numerical Methods in Engineering 1997; 40(11):2063-2086.

[3] Lyamin AV, Sloan SW. Upper bound limit analysis using linear finite elements and non-linear programming. International Journal for Numerical and Analytical Methods in Geomechanics 2002; 26(2):181216.

[4] Ciria H, Peraire J, Bonet J. Mesh adaptive computation of upper and lower bounds in limit analysis. International journal for numerical methods in engineering 2008; 75(8):899-944. 
[5] Gilbert M, He L, Smith CC, Le CV. Automatic yield-line analysis of slabs using discontinuity layout optimization. Proceedings of the Royal Society A: Mathematical, Physical and Engineering Science 2014; 470(2168):20140 071.

[6] Johansen K. Yield-line theory. Cement and Concrete Association London, 1962.

[7] Sloan SW, Kleeman PW. Upper bound limit analysis using discontinuous velocity fields. Computer Methods in Applied Mechanics and Engineering 1995; 127(1-4):293 - 314.

[8] Krabbenhoft K, Lyamin AV, Hjiaj M, Sloan SW. A new discontinuous upper bound limit analysis formulation. International Journal for Numerical Methods in Engineering 2005; 63(7):1069-1088.

[9] Makrodimopoulos A, Martin C. Upper bound limit analysis using discontinuous quadratic displacement fields. Communications in Numerical Methods in Engineering 2008; 24(11):911-927.

[10] Bleyer J, de Buhan P. On the performance of non-conforming finite elements for the upper bound limit analysis of plates. International Journal for Numerical Methods in Engineering 2013; 94(3):308-330, doi:10.1002/nme.4460.

[11] Zienkiewicz O, Taylor R, Too J. Reduced integration technique in general analysis of plates and shells. International Journal for Numerical Methods in Engineering 1971; 3(2):275-290.

[12] Hughes TJ, Taylor RL, Kanoknukulchai W. A simple and efficient finite element for plate bending. International Journal for Numerical Methods in Engineering 1977; 11(10):1529-1543.

[13] Lee S, Wong S. Mixed formulation finite elements for Mindlin theory plate bending. International Journal for Numerical Methods in Engineering 1982; 18(9):1297-1311.

[14] Zienkiewicz O, Lefebvre D. A robust triangular plate bending element of the Reissner-Mindlin type. International journal for numerical methods in engineering 1988; 26(5):1169-1184.

[15] Simo JC, Rifai M. A class of mixed assumed strain methods and the method of incompatible modes. International Journal for Numerical Methods in Engineering 1990; 29(8):1595-1638.

[16] Bathe KJ, Dvorkin EN. A four-node plate bending element based on Mindlin/Reissner plate theory and a mixed interpolation. International Journal for Numerical Methods in Engineering 1985; 21(2):367383.

[17] Bletzinger KU, Bischoff M, Ramm E. A unified approach for shear-locking-free triangular and rectangular shell finite elements. Computers \& Structures 2000; 75(3):321-334.

[18] Le CV. A stabilized discrete shear gap finite element for adaptive limit analysis of MindlinReissner plates. International Journal for Numerical Methods in Engineering 2013; 96:231-246, doi: $10.1002 /$ nme.4560.

[19] Salençon J. Yield Design. Wiley. com, 2013.

[20] Salençon J. Handbook of Continuum Mechanics: General Concepts, Thermoelasticity. Physics and Astronomy Online Library, Springer, 2001.

[21] Drucker D. The effect of shear on the plastic bending of beams. Journal of Applied Mechanics 1956; 23(4):509-514.

[22] Bleyer J, de Buhan P. Lower bound static approach for the yield design of thick plates. International Journal for Numerical Methods in Engineering 2014; 100(11):814-833.

[23] Papadopoulos P, Taylor RL. An analysis of inelastic Reissner-Mindlin plates. Finite elements in analysis and design 1991; 10(3):221-233.

[24] Ibrahimbegović A, Frey F. An efficient implementation of stress resultant plasticity in analysis of Reissner-Mindlin plates. International Journal for Numerical Methods in Engineering 1993; 36(2):303320 .

[25] Lubliner J. Plasticity Theory. Dover books on engineering, Dover Publications, 2008.

[26] Capsoni A, Corradi L. Limit analysis of plates- a finite element formulation. Structural Engineering and Mechanics $1999 ; \mathbf{8}(4): 325-341$.

[27] Makrodimopoulos A, Martin CM. Upper bound limit analysis using simplex strain elements and secondorder cone programming. International journal for numerical and analytical methods in geomechanics 2007; 31(6):835-865.

[28] Arnold DN. Discretization by finite elements of a model parameter dependent problem. Numerische Mathematik 1981; 37(3):405-421. 
[29] Arnold DN, Brezzi F, Falk RS, Marini LD. Locking-free Reissner-Mindlin elements without reduced integration. Computer methods in applied mechanics and engineering 2007; 196(37):3660-3671.

[30] Capsoni A, Vicente da Silva M. A finite element formulation of Mindlin plates for limit analysis. International Journal for Numerical Methods in Biomedical Engineering 2011; 27(1):143-156.

[31] Bræstrup M. Yield-line theory and limit analysis of plates and slabs. Danmarks Tekniske Højskole, Afdelingen for Bærende Konstruktioner, 1971.

[32] Hodge Jr PG, Belytschko T. Numerical methods for the limit analysis of plates. Journal of Applied Mechanics 1968; 35:796.

[33] Geuzaine C, Remacle JF. Gmsh: A 3-d finite element mesh generator with built-in pre-and postprocessing facilities. International Journal for Numerical Methods in Engineering 2009; 79(11):13091331.

[34] Mosek. The Mosek optimization toolbox for Matlab manual 2008. URL http://www.mosek.com/. 